\title{
THE FINITE ELEMENT METHOD FOR THE ELECTRIC FIELD OF MAXWELL'S BOUNDARY VALUE PROBLEM IN POLYGONAL DOMAINS OF PLANE
}

\author{
JUKKA SARANEN
}

Let $\Omega$ be a bounded polygonal domain in the plane $R^{2}$. The boundary $\Gamma$ is composed of a smooth part $\Gamma_{0}$ and of the vertices $\left\{a_{j}\right\}_{1}^{m}$. We study a finite element approximation process for Maxwell's boundary value problem

$$
\left\{\begin{array}{l}
\nabla \times \nabla \times E-\lambda E=F, \quad \lambda \neq 0, \quad \lambda \in \boldsymbol{R} \\
n \times\left. E\right|_{\Gamma_{0}}=0 .
\end{array}\right.
$$

Here $E$ and $F$ are fields over $\Omega$ and $n$ denotes the outer unit normal in the points of $\Gamma_{0}$. The above equation is satisfied by an electric field in a cylinder-type infinite spatial domain where the fields are independent of one of the coordinates. The study of the finite element approximation for Maxwell's boundary value problem was begun by Saranen in [12] with a discussion of a more general problem covering the anisotropic inhomogeneous case in smooth domains. Neittaanmäki and Picard [10] generalize the discussion to cover the pair $(E, H)$ of the electric field $E$ and magnetic field $H$. As regards the approximation of elliptic equations in the case of nonsmooth domains with the difference method or with finite elements, see Laasonen [5], Babuška [1] and Babuška-Rosenzweig [2]. In these papers a second order elliptic equation was considered. A general review can be found in StrangFix [16]. The plate equation is discussed in Melzer-Rannacher [9] and Stephan [15]. A useful tool in singular domains is a prior knowledge of the behavior of the solution in a neighborhood of singular boundary points. A highly significant - and frequently used - work in this respect is the article of Kondrat'ev [4] on general elliptic equations. However, the equation (0.1) represents a nonelliptic system, and the asymptotic of the solution near the corners is not known. This problem is considered first. Our trial subspaces consist of some piecewise linear continuous fields to which appropriate singular elements in the corners are added. Thus, a convergence

$$
\begin{aligned}
& h^{-1 / 2}\left\|E-E^{h}\right\|+\left\|\nabla \times\left(E-E^{h}\right)\right\|+\left\|\operatorname{div}\left(E-E^{h}\right)\right\| \\
& \leqq c h\left(\|F\|+\|\nabla \times F\|+\|\operatorname{div} F\|_{1}+\|\Delta \operatorname{div} F\|\right)
\end{aligned}
$$

with $c=c(\lambda)$ is achieved. 


\section{Continuous problem}

We put the problem (0.1) into the usual variational frame. All the functions and fields are real valued. The notations used for testfields, Sobolev spaces and for square-integrable fields are $\mathscr{D}(\Omega)^{2}=\mathscr{D}(\Omega) \times \mathscr{D}(\Omega), \mathscr{D}(\Omega)=C_{0}^{\infty}(\Omega), H^{k}(\Omega)^{2}=$ $H^{k}(\Omega) \times H^{k}(\Omega), L^{2}(\Omega)^{2}=L^{2}(\Omega) \times L^{2}(\Omega)$. The space $L^{2}(\Omega)^{2}$ is endowed with the scalar product

$$
(E \mid F)=\int_{\Omega} E \cdot F d x=\sum_{i=1}^{2}\left(E_{i} \mid F_{i}\right)
$$

with the corresponding norm $\|\cdot\|$. Similarly the Sobolev-norms $\|\cdot\|_{k}$ are given through the components. In $H^{1}(\Omega)^{2}, H^{1}(\Omega)$ the curl and divergence are given by $\nabla \times E=\partial_{1} E_{2}-\partial_{2} E_{1}, \quad \operatorname{div} E=\partial_{1} E_{1}+\partial_{2} E_{2}, \quad E \in H^{1}(\Omega)^{2}, \quad \nabla \times H=\left(\partial_{2} H,-\partial_{1} H\right)$, $H \in H^{1}(\Omega)$. These operations are defined in a weak sense ([6], [7], [18]) as well; put

$$
\begin{aligned}
& R(\Omega)=\left\{E \in L^{2}(\Omega)^{2} \mid \exists F_{1} \in L^{2}(\Omega):(\nabla \times \Phi \mid E)=\left(\Phi \mid F_{1}\right) \text { for every } \Phi \in \mathscr{D}(\Omega)\right\}, \\
& D(\Omega)=\left\{E \in L^{2}(\Omega)^{2} \mid \exists F_{2} \in L^{2}\{\Omega):(\nabla \Phi \mid E)=-\left(\Phi \mid F_{2}\right) \text { for every } \Phi \in \mathscr{D}(\Omega)\right\}
\end{aligned}
$$

and define $\nabla \times E=F_{1}, E \in R(\Omega)$, div $E=F_{2}, E \in D(\Omega)$. In $R(\Omega)$ the scalar product $(E \mid F)_{r}=(\nabla \times E \mid \nabla \times F)+(E \mid F)$ is also used, with the corresponding norm $\|\cdot\|_{r}$. The space $R^{0}(\Omega)=\overline{\mathscr{D}(\Omega)}\|\cdot\|_{r}$ characterizes the boundary condition $n \times\left. E\right|_{\Gamma_{0}}=0$. The imbedding $R^{0}(\Omega) \subset R(\Omega)$ is obvious.

1.1. Definition. Let $F \in D(\Omega)$. The field $E \in R^{0}(\Omega) \cap D(\Omega)$ is a solution of the problem $(0.1)$ if

$$
(\nabla \times \Phi \mid \nabla \times E)-\lambda(\Phi \mid E)=(\Phi \mid F)
$$

for every $\Phi \in R^{0}(\Omega)$.

The theory of existence for this problem is well developed. For example, the Fredholm property has been proved for a large class of domains ([7], [17]). The approximation of solution $E$ will be considered, when the right hand side $F$ satisfies the auxiliary conditions $F \in R(\Omega)$, $\operatorname{div} F \in H_{0}^{1}(\Omega)$. The latter condition enables an "elliptization" of the problem ([6], [7]). Denote $B(a, r)=\{x|| x-a \mid<r\}$, $U_{j}(r)=B\left(a_{j}, r\right) \cap \Omega$. In the following the radius $r$ is taken so small that the sets $U_{j}(2 r)$ are disjoint. Write $\Omega(r)=\Omega \backslash \bigcup_{j=1}^{m} \overline{U_{j}(r)}$. The notation $C$ refers to a generic constant independent on the fields under discussion. In some cases emphasis will be laid on its dependence on $\lambda, r$ etc. as $C(\lambda)$ etc.

1.2. Lemma. If $E$ is a solution of (0.1) such that $\operatorname{div} F \in H_{0}^{1}(\Omega)$ then $E \in H^{2}(\Omega(r))^{2}$ and

$$
\|E\|_{2, \Omega(r)} \leqq C(r, \lambda)\left(\|E\|+\|F\|+\|\operatorname{div} F\|_{1, \Omega}\right) .
$$


Proof. The equation (1.1) implies $\operatorname{div} E \in H_{0}^{1}(\Omega)$ with $\operatorname{div} E=-\lambda^{-1} \operatorname{div} F$. For $\Phi \in R^{0}(\Omega) \cap D(\Omega)$ we get

$$
\begin{aligned}
& (\nabla \times \Phi \mid \nabla \times E)+(\operatorname{div} \Phi \mid \operatorname{div} E)-\lambda(\Phi \mid E) \\
& =(\Phi \mid F)-\lambda^{-1}(\operatorname{div} \Phi \mid \operatorname{div} F)=(\Phi \mid G)
\end{aligned}
$$

with $G=F+\lambda^{-1} \nabla \operatorname{div} F \in L^{2}(\Omega)^{2}$. For smooth domains $\Omega_{0}$ the equation (1.3) yields the regularity $E \in H^{2}\left(\Omega_{0}\right)^{2}$ with

$$
\|E\|_{2, \Omega_{0}} \leqq C(\lambda)\left(\|E\|_{0, \Omega_{0}}+\|G\|_{0, \Omega_{0}}\right),
$$

([8], [11]). The estimate (1.2) follows with a standard smoothing and utilizing the equivalency of the norms $\|E\|_{1}$ and $\|E\|_{r+d}=\left(\|E\|_{r}^{2}+\|\operatorname{div} E\|^{2}\right)^{1 / 2}$ in $R^{0}\left(\Omega_{0}\right) \cap$ $D\left(\Omega_{0}\right)([7])$.

The behavior of the solution in a neighborhood of a corner $a_{j}$ will be discussed. The three dimensional case was considered in [13] and [14]. It is sufficient to choose $a_{j}=0$. For some $R>0,0<\alpha<2 \pi$ we have $U(R)=\{x \in \Omega|| x \mid<R\}=$ $\{x=x(r, \varphi) \mid 0<r<R, 0<\varphi<\alpha\}$, where $r, \varphi$ are the usual polar coordinates. Let us develop the fields $E$ and $F$ in $U(R)$ as follows. We write $E(r, \varphi)=E^{r}(r, \varphi) \bar{e}_{r}+$ $E^{\varphi}(r, \varphi) \bar{e}_{\varphi}$. Since $E \in L^{2}(U(R))^{2}$ the relation $E^{r}(r, \varphi), E^{\varphi}(r, \varphi) \in L^{2}((0, \alpha))$ holds for almost all $0<r<R$. Let $v_{n}(\varphi), w_{n}(\varphi)$ denote the orthonormal eigenfunctions for the Dirichlet and the Neumann problem $u^{\prime \prime}+\lambda u=0$ in $(0, \alpha) ; v_{n}(\varphi)=$ $\sqrt{2 / \alpha} \sin (n(\pi / \alpha) \varphi), \quad w_{n}(\varphi)=\sqrt{2 / \alpha} \cos (n(\pi / \alpha) \varphi), \quad n \geqq 1, \quad w_{0}(\varphi)=1 / \sqrt{\alpha}$. The corresponding eigenvalues are $\lambda_{n}=(n(\pi / \alpha))^{2}, n \geqq 1, \lambda_{0}=0$. For almost all $r$ the expansions

$$
E^{r}(r, \varphi)=\sum_{n=1}^{\infty} a_{n}(r) v_{n}(\varphi), \quad E^{\varphi}(r, \varphi)=\sum_{n=0}^{\infty} b_{n}(r) w_{n}(\varphi)
$$

hold in $L^{2}((0, \alpha))$. Because of $E \in L^{2}(U(R))^{2}$ it can be verified that

$$
E(r, \varphi)=\sum_{n=1}^{\infty} a_{n}(r) v_{n}(\varphi) \bar{e}_{r}+\sum_{n=0}^{\infty} b_{n}(r) w_{n}(\varphi) \bar{e}_{\varphi}
$$

converges in $L^{2}(U(R))^{2}$ with

$$
\|E\|_{0, U(R)}^{2}=\int_{0}^{R} r\left|b_{0}\right|^{2} d r+\sum_{n=1}^{\infty} \int_{0}^{R} r\left(\left|a_{n}\right|^{2}+\left|b_{n}\right|^{2}\right) d r .
$$

The field $F$ is similarly developed as

$$
F(r, \varphi)=\sum_{n=1}^{\infty} \alpha_{n}(r) v_{n}(\varphi) \bar{e}_{r}+\sum_{n=0}^{\infty} \beta_{n}(r) w_{n}(\varphi) \bar{e}_{\varphi}
$$

The equation $(0.1)$ is considered for a moment in the case where the fields have no sources, $\operatorname{div} E=\operatorname{div} F=0$. Because of $\Delta E=-\nabla \times \nabla \times E+\nabla \operatorname{div} E$ the equation $\Delta E+\lambda E=-F$ is distributionally satisfied. The vectorial Laplacian has the 
(distributional) representation

$$
\begin{aligned}
\Delta E & =\left(\Delta E^{r}-r^{-2} E^{r}-2 r^{-2} \partial_{\varphi} E^{r}\right) \bar{e}_{r} \\
& +\left(\Delta E^{\varphi}-r^{-2} E^{\varphi}+2 r^{-2} \partial_{\varphi} E^{r}\right) \bar{e}_{\varphi}
\end{aligned}
$$

in polar coordinates, and for the divergence we can calculate the expression

$$
\operatorname{div} E=\partial_{r} E^{r}+r^{-1} E^{r}+r^{-1} \partial_{\varphi} E^{\varphi} .
$$

The equation $\Delta E=-F-\lambda E, E \in R^{0}(\Omega) \cap D(\Omega)$ implies

$$
\begin{aligned}
& a_{n}^{\prime \prime}+r^{-1} a_{n}^{\prime}-r^{-2}\left(1+\lambda_{n}\right) a_{n}+2 r^{-2} \sqrt{\lambda_{n}} b_{n} \\
& =-\left(\alpha_{n}+\lambda a_{n}\right), \quad n \in N, \\
& b_{n}^{\prime \prime}+r^{-1} b_{n}^{\prime}-r^{-2}\left(1+\lambda_{n}\right) b_{n}+2 r^{-2} \sqrt{\lambda_{n}} a_{n} \\
& =-\left(\beta_{n}+\lambda b_{n}\right), \quad n \in N_{0}=N \cup\{0\}
\end{aligned}
$$

(define $a_{0}=0$ ). The condition $\operatorname{div} E=0$ leads to

$$
a_{n}^{\prime}+r^{-1} a_{n}-r^{-1} \sqrt{\lambda_{n}} b_{n}=0, \quad n \in N .
$$

These equations are distributionally satisfied and the coefficients have the regularity $a_{n}, b_{n} \in H_{\mathrm{loc}}^{2}((0, R))$. For the rotation one obtains

$$
\nabla \times E=\partial_{r} E^{\varphi}-r^{-1} \partial_{\varphi} E^{r}
$$

and therefore

$$
\|\nabla \times E\|_{0, U(R)}^{2}=\int_{0}^{R} r\left|b_{0}^{\prime}\right|^{2} d r+\sum_{n=1}^{\infty} \int_{0}^{R} r\left|b_{n}^{\prime}-r^{-1} \sqrt{\lambda_{n}} a_{n}\right|^{2} d r .
$$

We decompose the solution into a singular part and into another belonging to $H^{2}(\Omega)^{2}$. This is carried out by application of Kondrat'ev's method to the differential equations (1.10) and (1.11). Thus we obtain an appropriate decomposition for the coefficients $a_{n}, b_{n}$. The three dimensional case was handled similarly in [14]. Some notations will be needed in the following proof. Denote

$$
\begin{array}{ll}
L_{n} u=-\left(u^{\prime \prime}+3 r^{-1} u^{\prime}+r^{-2}\left(1-\lambda_{n}\right) u\right), & n \in N, \\
S_{n} u=-\left(u^{\prime \prime}+r^{-1} u^{\prime}-r^{-2} \lambda_{n} u\right), & n \in N, \\
S_{0} u=-\left(u^{\prime \prime}+r^{-1} u^{\prime}-r^{-2} u\right) . &
\end{array}
$$

Let the spaces $W_{\alpha}^{k}(R), R>0$ be introduced with the norms $\|\cdot\|_{k, \alpha}(R)$ such that

$$
\begin{aligned}
\|u\|_{k, \alpha}^{2}(R) & =\sum_{v=0}^{k} \int_{0}^{R} r^{\alpha-2 v}\left|\left(\frac{d}{d r}\right)^{v} u(r)\right|^{2} d r \\
& =\sum_{v=0}^{k}\left\|r^{\alpha / 2-v} u^{(v)}(r)\right\|^{2}(R),
\end{aligned}
$$


$\|u\|(R):=\|u\|_{0, U(R)}$, and that $W_{\alpha}^{k}(R)=\left\{u \in H_{\mathrm{loc}}^{k}(0, R) \mid\|u\|_{k, \alpha}(R)<\infty\right\}$. Further, the norms $\|\cdot\|_{k, \alpha,\left(\lambda_{n}\right)}(R)$

will be used.

$$
\|u\|_{k, \alpha,\left(\lambda_{n}\right)}^{2}(R)=\sum_{\nu=0}^{k} \lambda_{n}^{k-v}\left\|r^{\alpha / 2-v} u^{(v)}(r)\right\|^{2}(R)
$$

1.3. Theorem. Suppose that $\alpha \neq k(\pi / 2)$. For the solution $E \in R^{0}(\Omega) \cap D(\Omega)$ of (1.1) with $F \in R(\Omega) \cap D(\Omega)$, div $F \in H_{0}^{1}(\Omega)$ in $U\left(R_{0}\right), 0<R_{0}<R$ the decomposition

$$
E=\sum_{0<n \frac{\pi}{\alpha}<2} r^{-1+n(\pi / \alpha)}\left(\delta_{n}^{1} v_{n}(\varphi) \bar{e}_{r}+\delta_{n}^{2} w_{n}(\varphi) \bar{e}_{\varphi}\right)+\delta_{0}^{2} r \bar{e}_{\varphi}+W
$$

with $W \in H^{2}\left(U\left(R_{0}\right)\right)^{2}$ and with the constants $\delta_{n}^{i}$ such that

holds.

$$
\begin{aligned}
& \|W\|_{2, U\left(R_{0}\right)}^{2}+\delta_{0}^{2}+\sum_{0<n \frac{\pi}{\alpha}<2}\left(\left(\delta_{n}^{1}\right)^{2}+\left(\delta_{n}^{2}\right)^{2}\right) \\
& \leqq C(\lambda)\left(\|E\|^{2}+\|F\|_{r}^{2}+\|\operatorname{div} F\|_{1}^{2}\right)
\end{aligned}
$$

Proof. We apply the usual decomposition $F=F_{0}+F_{1}$ of the field $F$ into a sourcefree part $F_{0}$ and into another $F_{1} \in \nabla H_{0}^{1}(\Omega) ; F_{1}=\nabla \Delta_{0}^{-1}(\operatorname{div} F)$ ([7]). Here $\Delta_{0}$ denotes the Laplacian with the homogeneous Dirichlet boundary condition defined in $D\left(\Delta_{0}\right)=\left\{u \in H_{0}^{1}(\Omega) \mid \Delta u \in L^{2}(\Omega)\right\}$. If the solution $E=E_{0}+E_{1}$ is analogously decomposed, $E_{1}=-i^{-1} F_{1}$ from (1.1) is obtained. Since $\operatorname{div} F \in H_{0}^{1}(\Omega)$ the equation $\Delta_{0} u=-i^{-1} \operatorname{div} F$ implies in $U\left(R_{0}\right)$

$$
u=\sum_{0<\frac{n \pi}{x}<2} \gamma_{n} r^{n(\pi / x)} v_{n}(\varphi)+w,
$$

where $w \in H^{3}\left(U\left(R_{0}\right)\right)$ and where

$$
\gamma_{n}=\frac{\alpha}{2 n \pi} \int_{0}^{R} r^{1-n(\pi / x)} S_{n}\left(\eta c_{n}\right) d r
$$

Further,

$$
\|w\|_{3, U\left(R_{0}\right)}^{2}+\sum_{0<\frac{n \pi}{\alpha}<2} \gamma_{n}^{2} \leqq C(\lambda)\|\operatorname{div} F\|_{1, \Omega}^{2}
$$

[14, Lemma 3.2]. In the formula (1.18) $\eta$ is an arbitrary function $\eta \in \mathscr{D}(-\infty, R)$ such that $\eta(r) \equiv 1$ in a neighborhood of $r=0$. The functions $c_{n}$ are the coefficients in the expansion $u(r, \varphi)=\sum_{n=1}^{\infty} c_{n}(r) v_{n}(\varphi)$. It follows from (1.17) and (1.19) that the part $E_{1}=\nabla u$ satisfies the decomposition of (1.15) with (1.16). Accordingly, the case $\operatorname{div} E=\operatorname{div} F=0$ can be considered. The equations (1.10i) and (1.11) imply

$$
L_{n} a_{n}=\alpha_{n}+\lambda a_{n}, \quad n \in N .
$$

Fix a number $R_{1}, R_{0}<R_{1}<R$ and $\eta \in \mathscr{D}(\boldsymbol{R})$ with $\eta(r) \equiv 1,0 \leqq r \leqq R_{0}, \eta(r)=0$, $r \geqq R_{1}$. The relations (1.6), (1.11) and (1.20) give $\eta a_{n} \in W_{5}^{2}\left(R_{1}\right)$. Since $L_{n}\left(\eta a_{n}\right) \in W_{1}^{0}\left(R_{1}\right)$ 
and since $n(\pi / \alpha) \neq 2$ for every $n$, Lemma 2.1 can be applied (see also Satz 2.2 in [14]). This yields

$$
a_{n}(r)=a_{n}^{0}(r)+\sigma_{n}^{1} r^{-1+n(\pi / \alpha)}
$$

in $\left(0, R_{0}\right)$ with $a_{n}^{0} \in W_{1}^{2}\left(R_{0}\right)$

$$
\left\|a_{n}^{0}\right\|_{2,1,\left(\lambda_{n}\right)}\left(R_{0}\right) \leqq C\left\|L_{n}\left(\eta a_{n}\right)\right\|_{0,1}\left(R_{1}\right)
$$

and with

$$
\sigma_{n}^{1}=\frac{\alpha}{2 n \pi} \int_{0}^{R_{1}} r^{2-n(\pi / \alpha)} L_{n}\left(\eta a_{n}\right) d r, \quad \frac{n \pi}{\alpha}<2
$$

$\sigma_{n}^{1}=0, n \pi / \alpha>2$. From (1.22) we can conclude further

$$
\begin{aligned}
& \left\|a_{n}^{0}\right\|_{2,1,\left(\lambda_{n}\right)}\left(R_{0}\right) \\
& \leqq C(\lambda)\left(\left\|r^{1 / 2} \alpha_{n}\right\|\left(R_{1}\right)+\left\|r^{1 / 2} a_{n}\right\|\left(R_{1}\right)+\left\|a_{n}\right\|_{1,\left(R_{0}, R_{1}\right)}\right) \\
& \leqq C(\lambda)\left(\left\|r^{1 / 2} \alpha_{n}\right\|\left(R_{1}\right)+\left\|r^{1 / 2} a_{n}\right\|\left(R_{1}\right)+\sqrt{\lambda_{n}}\left\|r^{1 / 2} b_{n}\right\|\left(R_{1}\right)\right) .
\end{aligned}
$$

It is enough to consider the values $n(\pi / \alpha)>2$. For those indices

$$
\begin{aligned}
& \left\|a_{n}\right\|_{2,1,\left(\lambda_{n}\right)}\left(R_{0}\right) \\
& \leqq C(\lambda)\left(\left\|r^{1 / 2} \alpha_{n}\right\|\left(R_{1}\right)+\left\|r^{1 / 2} a_{n}\right\|\left(R_{1}\right)+\left\|a_{n}\right\|_{1,\left(R_{0}, R_{1}\right)}\right)
\end{aligned}
$$

is obtained from (1.24). This inequality holds in the same way for the pair $R_{1}, R$ as for $R_{0}, R_{1}$. Thus

$$
\begin{aligned}
& \left\|a_{n}\right\|_{1,\left(R_{0}, R_{1}\right)} \leqq C(\lambda) \lambda_{n}^{-1 / 2}\left\|a_{n}\right\|_{2,1,\left(\lambda_{n}\right)}\left(R_{1}\right) \\
& \leqq C(\lambda)\left(\left\|r^{1 / 2} \alpha_{n}\right\|(R)+\left\|r^{1 / 2} a_{n}\right\|(R)+\left\|r^{1 / 2} b_{n}\right\|(R)\right) .
\end{aligned}
$$

From (1.25) and (1.24) follows

$$
\begin{aligned}
& \left\|a_{n}^{0}\right\|_{2,1,\left(\lambda_{n}\right)}\left(R_{0}\right) \\
& \leqq C(\lambda)\left(\left\|r^{1 / 2} \alpha_{n}\right\|(R)+\left\|r^{1 / 2} a_{n}\right\|(R)+\left\|r^{1 / 2} b_{n}\right\|(R)\right) .
\end{aligned}
$$

The same bound can be found for $\sigma_{n}^{1}$ from (1.23). For the indices $n \geqq 1$ the coefficients $b_{n}$ are decomposed through formula (1.11) as

$$
b_{n}=b_{n}^{0}+\sigma_{n}^{2} r^{1-n(\pi / \alpha)} \text {, }
$$

where

$$
\begin{aligned}
& b_{n}^{0}=\frac{\alpha}{n \pi}\left(r\left(a_{n}^{0}\right)^{\prime}+a_{n}^{0}\right), \\
& \sigma_{n}^{2}=\frac{\alpha}{n \pi}\left(1+\frac{n \pi}{\alpha}\right) \sigma_{n}^{1} .
\end{aligned}
$$

Utilizing (1.27) and (1.10ii) we can verify

$$
\begin{aligned}
& \left\|b_{n}^{0}\right\|_{2,1,\left(\lambda_{n}\right)}\left(R_{0}\right) \\
& \leqq C(\lambda)\left(\left\|r^{1 / 2} \alpha_{n}\right\|(R)+\left\|r^{1 / 2} \beta_{n}\right\|(R)+\left\|r^{1 / 2} a_{n}\right\|(R)+\left\|r^{1 / 2} b_{n}\right\|(R)\right) .
\end{aligned}
$$


The coefficient $b_{0}$ must be handled in a different way. The assumption $\nabla \times F \in L^{2}(\Omega)$ will be used here. Indeed, this implies ((1.13))

$$
\int_{0}^{R} r\left|\beta_{0}^{\prime}\right|^{2} d r \leqq\|\nabla \times F\|^{2}<\infty .
$$

Choose $\varepsilon, 0<\varepsilon<1$. According to [4, Lemma 4.4],

$$
\begin{aligned}
& \int_{0}^{R} r^{-1+2 \varepsilon}\left|\eta \beta_{0}\right|^{2} d r \leqq \varepsilon^{-2} \int_{0}^{R} r^{1+2 \varepsilon}\left|\left(\eta \beta_{0}\right)^{\prime}\right|^{2} d r \\
& \leqq C\left(\int_{0}^{R} r^{1+2 \varepsilon}\left|\beta_{0}^{\prime}\right|^{2} d r+\int_{0}^{R} r\left|\beta_{0}\right|^{2} d r\right) \\
& \leqq C\left(\|\nabla \times F\|^{2}+\|F\|^{2}\right)
\end{aligned}
$$

can be calculated with $C=C(\varepsilon)$. We estimate $\eta b_{0}$ in the same way. Then

$$
b_{0}^{\prime \prime}+r^{-1} b_{0}^{\prime}-r^{-2} b_{0}=-\left(\beta_{0}+\lambda b_{0}\right)
$$

$\eta b_{0} \in W_{3+2 \varepsilon}^{2}(R), S_{0}\left(\eta b_{0}\right) \in W_{-1+2 \varepsilon}^{0}(R)$ is concluded from equation (1.10ii). Applying [14, Lemma 2.1] again we obtain

$$
b_{0}(r)=b_{0}^{0}(r)+\sigma_{0}^{2} r
$$

in $\left(0, R_{n}\right)$ with

$$
\begin{aligned}
\left\|b_{0}^{0}\right\|_{2,1}\left(R_{0}\right) & \leqq C\left\|b_{0}^{0}\right\|_{2,1-2 \varepsilon}\left(R_{0}\right) \leqq C\left\|S_{0}\left(\eta b_{0}\right)\right\|_{0,1-2 \varepsilon}(R) \\
& \leqq C(\lambda)(\|\nabla \times F\|+\|F\|+\|E\|) .
\end{aligned}
$$

The coefficient $\sigma_{0}^{2}$ has the form

$$
\sigma_{0}^{2}=\frac{1}{2} \int_{0}^{R} S_{0}\left(\eta b_{0}\right) d r
$$

and it can be estimated with the bound in (1.34). The field $E$ takes the representation

$$
\begin{aligned}
& E(r, \varphi) \\
& =\sum_{0<n \frac{\pi}{\alpha}<2} r^{-1+n(\pi / \alpha)}\left(\sigma_{n}^{1} v_{n}(\varphi) \bar{e}_{r}+\sigma_{n}^{2} w_{n}(\varphi) \bar{e}_{\varphi}\right)+\sigma_{0}^{2} r \bar{e}_{\varphi}+W
\end{aligned}
$$

in $L^{2}\left(U\left(R_{0}\right)\right)^{2}$ with

$$
W=\sum_{n=1}^{\infty} a_{n}^{0} v_{n} \bar{e}_{r}+\sum_{n=0}^{\infty} b_{n}^{0} w_{n} \bar{e}_{\varphi}
$$

The expansion (1.37) converges in $L^{2}\left(U\left(R_{0}\right)\right)^{2}$ because $a_{n}^{0}=a_{n}, b_{n}^{0}=b_{n}, n(\pi / \alpha)>2$. 
It remains to analyse the convergence of (1.37) with respect to the $\|\cdot\|_{2, U\left(R_{0}\right)}$ norm. Denote

$$
S_{N}=\sum_{n=1}^{N} a_{n}^{0} v_{n} \bar{e}_{r}+\sum_{n=0}^{N} b_{n}^{0} w_{n} \bar{e}_{\varphi}
$$

The estimate

$$
\begin{aligned}
& \left\|S_{N}\right\|_{2, U\left(R_{0}\right)}^{2} \\
& \leqq C\left(\left\|b_{0}^{0}\right\|_{2,1}^{2}\left(R_{0}\right)+\sum_{n=1}^{N}\left(\left\|a_{n}^{0}\right\|_{2,\left(\lambda_{n}\right)}^{2}\left(R_{0}\right)+\left\|b_{n}^{2}\right\|_{2,1,\left(\lambda_{n}\right)}^{2}\left(R_{0}\right)\right)\right)
\end{aligned}
$$

is verified. We have through (1.27), (1.31) and (1.34)

$$
\begin{aligned}
& \left\|b_{0}^{0}\right\|_{2,1}^{2}\left(R_{0}\right)+\sum_{n=1}^{\infty}\left(\left\|a_{n}^{0}\right\|_{2,1,\left(\lambda_{n}\right)}^{2}\left(R_{0}\right)+\left\|b_{n}^{0}\right\|_{2,1,\left(\lambda_{n}\right)}^{2}\left(R_{0}\right)\right) \\
& \leqq C(\lambda)\left(\|E\|^{2}+\|F\|^{2}+\|\nabla \times F\|^{2}\right)
\end{aligned}
$$

Thus, the series (1.37) converges with respect to the norm $\| \cdot{ }_{2, U\left(R_{0}\right)}$, and for the limit $W \in H^{2}\left(U\left(R_{0}\right)\right)^{2}$ the inequality

$$
\|W\|_{2, U\left(R_{0}\right)}^{2} \leqq C(\lambda)\left(\|E\|^{2}+\|F\|_{r}^{2}\right)
$$

is true.

A global decomposition can now be given. Fix polar coordinates in the neighborhood $U_{j}(2 R)$ of the corner $a_{j}$ and take $\xi \in \mathscr{D}(\boldsymbol{R})$ with $\xi(r) \equiv 1,0 \leqq r \leqq R_{0} / 2$, $\xi(r)=0, r \geqq R_{0}$. Define the singular fields

$$
\begin{aligned}
& S_{n, j}^{r}(x)=\left|x-a_{j}\right|^{-1+n\left(\pi / \alpha_{j}\right)} \xi\left(\left|x-a_{j}\right|\right) v_{n}^{j}\left(\varphi_{j}\right) \bar{e}_{r}^{j}, \\
& S_{n, j}^{\varphi}(x)=\left|x-a_{j}\right|^{-1+n\left(\pi / \alpha_{j}\right)} \xi\left(\left|x-a_{j}\right|\right) w_{n}^{j}\left(\varphi_{j}\right) \bar{e}_{\varphi}^{j}, \\
& S_{0, j}^{\varphi}(x)=\left|x-a_{j}\right| \xi\left(\left|x-a_{j}\right|\right) \bar{e}_{\varphi}^{j} .
\end{aligned}
$$

1.4. Theorem. Suppose that $\alpha_{j} \neq k(\pi / 2)$. The solution $E \in R^{0}(\Omega) \cap D(\Omega)$ of (1.1) with $F \in R(\Omega) \cap D(\Omega)$, div $F \in H_{0}^{1}(\Omega)$ has the decomposition

$$
E=\sum_{j=1}^{m}\left(\sum_{0<n \frac{\pi}{\alpha_{j}}<2}\left(\delta_{n, j}^{1} S_{n, j}^{r}+\delta_{n, j}^{2} S_{n, j}^{\varphi}\right)+\delta_{0, j}^{2} S_{0, j}^{\varphi}\right)+W
$$

with $W \in H^{2}(\Omega)^{2}$ and with the constants $\delta_{n, j}^{i}$ such that

$$
\begin{aligned}
& \|W\|_{2, \Omega}^{2}+\sum_{j=1}^{m}\left(\delta_{0, j}^{2}+\sum_{0<n \frac{\pi}{\alpha_{j}}<2}\left(\left(\delta_{n, j}^{1}\right)^{2}+\left(\delta_{n, j}^{2}\right)^{2}\right)\right) \\
& \leqq C(\lambda)\left(\|E\|^{2}+\|F\|_{r}^{2}+\|\operatorname{div} F\|_{1}^{2}\right) .
\end{aligned}
$$




\section{Finite element approximation}

The solution $E$ will be approximated by means of the bilinear form

$$
B_{\lambda}(\Phi, E)=(\nabla \times \Phi \mid \nabla \times E)+(\operatorname{div} \Phi \mid \operatorname{div} E)-\lambda(\Phi \mid E) .
$$

According to (1.3), $E$ satisfies the variational characterization

$$
B_{\lambda}(\Phi, E)=(\Phi \mid G), \quad \Phi \in R^{0}(\Omega) \cap D(\Omega)
$$

with $G=F+\lambda^{-1} \nabla \operatorname{div} F$. Let $\left(\mathscr{T}_{h}\right), 0<h \leqq h_{0}$ be a family of regular triangulations of the domain $\Omega$. By regularity we mean that there exist two constants $c_{0}, c_{1}>0$ such that every triangle $T_{h} \in \mathscr{T}_{h}$ contains a circular disc with the radius $c_{0} h$ and is contained within another disc with the radius $c_{1} h$. As usual $\bar{\Omega}=\cup\left\{\bar{T}_{h} \mid T_{h} \in \mathscr{T}_{h}\right\}$. Let $\mathscr{P}_{h}$ denote the set of knots of $\mathscr{T}_{h}$ lying on the boundary $\partial \Omega$. Note especially, that the vertices $a_{j}$ belong to $\mathscr{P}_{h}$. Define

$$
S^{h}=\left\{\Phi=\left(\Phi_{1}, \Phi_{2}\right) \in C(\bar{\Omega})^{2}\left|\Phi_{i}\right|_{T_{h}} \text { linear, }(n \times \Phi)(x)=0, x \in \mathscr{P}_{h} \cap \Gamma_{0}, \Phi\left(a_{j}\right)=0\right\} .{ }^{1)}
$$

Note that if $E$ is a solution satisfying $E \in H^{2}(\Omega)^{2}$, then the linear interpolate $J^{h} E$ belongs to $S^{h}$. The trial subspace which is to be used is defined by

$$
\tilde{S}^{h}=\left\{\psi=\sum_{j=1}^{m}\left(\sum_{0<n \frac{\pi}{\alpha_{j}}<2}\left(\alpha_{n, j} S_{n, j}^{r}+\beta_{n, j} S_{n, j}^{\varphi}\right)+\beta_{0, j} S_{0, j}^{\varphi}\right)+\Phi \mid \Phi \in S^{h}, \alpha_{n, j}, \beta_{n j} \in \boldsymbol{R}\right\}
$$

Observe that $\tilde{S}^{h} \subset R^{0}(\Omega) \cap D(\Omega)$. Hence the method is conform. For arbitrary smooth domains the situation is different, the use of $S^{h}$ leading to a non-conforming method. In such a case it is natural to utilize the equivalency of the $\|\cdot\|_{1}$ and $\|\cdot\|_{r, d}$-norms over $S^{h}$ ([12]). Here it is not necessary. The approximate solution $E^{h} \in \widetilde{S}^{h}$ is defined through

$$
B_{\lambda}\left(\psi, E^{h}\right)=(\psi \mid G), \quad \psi \in \tilde{S}^{h} .
$$

If $\lambda<0$, then (2.5) has a unique solution. In the case $\lambda>0$, when $\lambda$ is not an eigenvalue of (2.2), it turns out that the equation (2.5) has a unique solution for a sufficiently small $h$ (for a similar situation see [12]). Let us first consider the case $\lambda<0$. Owing to conformality one has the minimum characterization

$$
B_{\lambda}\left(E-E^{h}\right)=\min _{\psi \in \tilde{S}^{h}} B_{\lambda}(E-\psi)
$$

(see [16]). Here we have denoted $B_{\lambda}(\psi)=B_{\lambda}(\psi, \psi)$. For a solution $E=S+W$, where $S$ is the singular term, we use the "generalized interpolate" $\tilde{J}^{h} E=S+J^{h} W$.

1) $C(\bar{\Omega})^{2}$ refers to continuous fields over $\bar{\Omega}$. 
Because of $W \in H^{2}(\Omega)^{2}$

$$
\begin{aligned}
B_{\lambda}\left(E-E^{h}\right) & \leqq B_{\lambda}\left(E-\tilde{J}^{h} E\right)=B_{\lambda}\left(W-J^{h} W\right) \leqq C h^{2}\|W\|_{2, \Omega}^{2} \\
& \leqq C(\lambda) h^{2}\left(\|F\|_{r}^{2}+\|\operatorname{div} F\|_{1}^{2}\right)
\end{aligned}
$$

is obtained from (1.42). We shall now consider Nitsche's trick. As is known, the essential point there is to solve the equation $Z \in R^{0}(\Omega) \cap D(\Omega)$

$$
B_{\lambda}(\Phi, Z)=\left(\Phi \mid E-E^{h}\right), \quad \Phi \in R^{0}(\Omega) \cap D(\Omega) .
$$

Here this causes additional difficulties since our decomposition (1.41) was proved only for solutions $Z$ of the type

$$
(\nabla \times \Phi \mid \nabla \times Z)-\lambda(\Phi \mid Z)=(\Phi \mid F), \quad \Phi \in R^{0}(\Omega) \cap D(\Omega)
$$

(or equivalently $\Phi \in R^{0}(\Omega)$, since $R^{0}(\Omega) \cap D(\Omega)$ is dense in $R^{0}(\Omega)$ ) with $Z \in R^{0}(\Omega) \cap D(\Omega), F \in D(\Omega) \cap R(\Omega)$, div $F \in H_{0}^{1}(\Omega)$. However, because $Z$ is a solution of (2.8) it satisfies the regularity $\operatorname{div} Z \in H_{0}^{1}(\Omega)$ ([10]). Hence $Z$ satisfies (2.9) with $F=E-E^{h}+\nabla \operatorname{div} Z \in L^{2}(\Omega)^{2}$. Moreover, $\operatorname{div} F=\operatorname{div}\left(E-E^{h}\right)+\nabla \operatorname{div} Z=$ $-\lambda \operatorname{div} Z \in H_{0}^{1}(\Omega)$. Thus the decomposition (1.41) is applicable to $Z$ :

$$
Z=S_{1}+W_{1},
$$

where

$$
\begin{aligned}
\left\|W_{1}\right\|_{2} & \leqq C(\lambda)\left(\|Z\|+\left\|E-E^{h}\right\|+\left\|\nabla \times\left(E-E^{h}\right)\right\|+\|\operatorname{div} Z\|_{1}\right) \\
& \leqq C(\lambda)\left(\left\|E-E^{h}\right\|+\left\|\nabla \times\left(E-E^{h}\right)\right\|+\left\|\operatorname{div}\left(E-E^{h}\right)\right\|\right) .
\end{aligned}
$$

The last inequality follows from (2.8) and from $\left(\nabla_{0}+\lambda\right) \operatorname{div} Z=-\operatorname{div}\left(E-E^{h}\right)$. With an arbitrary field $\psi \in \widetilde{S}^{h}$ the inequality

$$
\left\|E-E^{h}\right\|^{2}=B_{\lambda}\left(E-E^{h}, Z-\psi\right) \leqq B_{\lambda}\left(E-E^{h}\right)^{1 / 2} B_{\lambda}(Z-\psi)^{1 / 2}
$$

holds. We choose $\psi=\tilde{J}^{h} Z=S_{1}+J^{h} W_{1}$. Then

$$
B_{\lambda}(Z-\psi)^{1 / 2} \leqq c h\left\|W_{1}\right\|_{2} .
$$

Using (2.7), (2.11)-(2.13) we obtain

$$
\begin{aligned}
\left\|E-E^{h}\right\|^{2} & \leqq C(\lambda) h^{2}\left(\|E\|+\|F\|_{r}+\|\operatorname{div} F\|_{1}\right)\left\|E-E^{h}\right\|_{r+d} \\
& \leqq C(\lambda) h^{3}\left(\|F\|_{r}+\|\operatorname{div} F\|_{1}\right)^{2} .
\end{aligned}
$$

Thus inequality (0.2) is proved.

The case $\lambda>0$, when $\lambda$ is not an eigenvalue of (2.2), can be handled by means of an inversion with a compact operator as in [12]. However, since in the estimates (2.7) and (2.14) more regularity than $L^{2}$ from the right side was needed, the sufficiency of the same conditions for the right side is not obvious. In fact the requirement $\Delta \operatorname{div} F \in L^{2}(\Omega)$ comes here into use. Let us formulate our result: 
2.1. Theorem. Assume that $\Omega \subset \boldsymbol{R}^{2}$ is a polygonal domain of the plane such that $\alpha_{j} \neq k(\pi / 2)$ for every $j$. If $E \in R^{0}(\Omega) \cap D(\Omega)$ is a solution of (1.1) with $F \in R(\Omega) \cap D(\Omega)$, $\operatorname{div} F \in H_{0}^{1}(\Omega), \Delta \operatorname{div} F \in L^{2}(\Omega)$ such that $\lambda(\neq 0)$ is no eigenvalue of (2.2), then the approximation $E^{h} \in \widetilde{S}^{h}$ defined by (2.5) gives the error estimate (0.2). The assumption $\Delta \operatorname{div} F \in L^{2}(\Omega)$ is not necessary if $\lambda<0$. Accordingly, the estimates (2.7), (2.14) are valid instead of (0.2).

Proof. Only the case $\lambda>0, \lambda$ being not an eigenvalue, needs an argumentation. Take a number $\mu<0$. Let $K_{\mu}: L^{2}(\Omega)^{2} \rightarrow R^{0}(\Omega) \cap D(\Omega)$ be the solution operator of the equation

$$
B_{\mu}(\Phi, E)=(\Phi \mid G), \quad \Phi \in R^{0}(\Omega) \cap D(\Omega)
$$

with $E=K_{\mu} G \in R^{0}(\Omega) \cap D(\Omega)$. Equivalently

$$
\left(I-(\lambda+\mu) K_{\mu}\right) E=K_{\mu} G, \quad E \in R^{0}(\Omega) \cap D(\Omega) .
$$

Since $K_{\mu}$ is compact in $L^{2}(\Omega)^{2}$ ([17]), one verifies that the restriction of $K_{\mu}$ to $R^{0}(\Omega) \cap D(\Omega)$ is compact, too (compare [12]). Equation (2.5) in turn is equivalent with

$$
\left(I-(\lambda+\mu) K_{\mu}^{h}\right) E^{h}=K_{\mu}^{h} G, \quad E^{h} \in R^{0}(\Omega) \cap D(\Omega),
$$

where $K_{\mu}^{h}$ denotes the solution operator of (2.5) with $\lambda=\mu$. Let us consider the approximation error with respect the $\|\cdot\|_{r+d}$ norm. First, the inequality

$$
\left\|\left(K_{\mu}-K_{\mu}^{h}\right) \tilde{G}\right\|_{r+d} \leqq c h\|\tilde{G}\|_{r+d}
$$

holds for $\tilde{G} \in R(\Omega) \cap D(\Omega)$. Indeed, take $G=\tilde{G}$ in (2.15) and denote $\widetilde{E}=K_{\mu} \widetilde{G}$, $\tilde{E}^{h}=K_{\mu}^{h} \widetilde{G}$. Then $(2.15)$ implies $\operatorname{div} \tilde{E} \in H_{0}^{1}(\Omega)$ and $\left(-\Delta_{0}+|\mu|\right) \operatorname{div} \tilde{E}=\operatorname{div} \tilde{G}$. Thus

$$
\|\operatorname{div} \tilde{E}\|_{1} \leqq c\|\operatorname{div} \tilde{G}\| .
$$

On the other hand, (2.12) leads to

$$
(\nabla \times \Phi \mid \nabla \times \widetilde{E})+|\mu|(\Phi \mid \widetilde{E})=(\Phi \mid \widetilde{F}), \quad \Phi \in R^{0}(\Omega)
$$

with $\tilde{F}=\widetilde{G}+\nabla \operatorname{div} \widetilde{E}, \operatorname{div} \widetilde{F}=\operatorname{div} \widetilde{G}+\Delta_{0} \operatorname{div} \widetilde{E}=|\mu| \operatorname{div} \tilde{E} \in H_{0}^{1}(\Omega), \quad \tilde{F} \in R(\Omega) \cap D(\Omega)$. According to (2.7), (2.19)

$$
\left\|\tilde{E}-\widetilde{E}^{h}\right\|_{r+d} \leqq \operatorname{ch}\left(\|\tilde{E}\|+\|\tilde{F}\|_{r}+\|\operatorname{div} \tilde{F}\|_{1}\right) \leqq c h\|\widetilde{G}\|_{r+d} .
$$

If $\|K\|_{r+d, \mathrm{r}+d}$ denotes the operator norm for operators $K:\left(R \cap D,\|\cdot\|_{\mathrm{r}+d}\right) \mapsto$ $\left(R \cap D,\|\cdot\|_{r+d}\right)$, then

$$
\left\|K_{\mu}-K_{\mu}^{h}\right\|_{r+d, r+d} \leqq c h
$$

follows from (2.18). Owing to compactness, the inverse $\left(I-(\lambda+\mu) K_{\mu}\right)^{-1}$ in $R^{0}(\Omega) \cap D(\Omega)$ exists, since $\lambda$ is no eigenvalue of (2.2). Accordingly, the inverse 
of $I-(\lambda+\mu) K_{\mu}^{h}$ also exists if $h$ is sufficiently small. Hence (2.5) is uniquely solvable for small $h$. Moreover, a Neumann expansion gives the estimate

$$
\left\|\left(I-(\lambda+\mu) K_{\mu}\right)^{-1}-\left(I-(\lambda+\mu) K_{\mu}^{h}\right)^{-1}\right\|_{\mid r+d, r+d} \leqq c h .
$$

We can now calculate

$$
\begin{aligned}
\left\|E-E^{h}\right\|_{r+d} \leqq & \left\|\left[\left(I-(\lambda+\mu) K_{\mu}\right)^{-1}-\left(I-(\lambda+\mu) K_{\mu}^{h}\right)^{-1}\right] K_{\mu} G\right\|_{r+d} \\
& +\left\|\left(I-(\lambda+\mu) K_{\mu}^{h}\right)^{-1}\left(K_{\mu}-K_{\mu}^{h}\right) G\right\|_{r+d} \\
\leqq & c h\left\|K_{\mu} G\right\|_{r+a}+c\left\|\left(K_{\mu}-K_{\mu}^{h}\right) G\right\|_{r+d} \\
\leqq & c h\|G\|_{r+d}+c\left\|\left(K_{\mu}-K_{\mu}^{h}\right) G\right\|_{r+d} \\
\leqq & c h\left(\|F\|+\|\operatorname{div} F\|_{1}\right)+c\left\|\left(K-K_{\mu}^{h}\right) G\right\|_{r+d} .
\end{aligned}
$$

For the estimation of the second term we make use of the assumption $\Delta \operatorname{div} F \in L^{2}(\Omega)$ This garantees that $G=F+\lambda^{-1} \nabla \operatorname{div} F \in R(\Omega) \cap D(\Omega)$. Hence inequality (2.18) is applicable with $\tilde{G}=G$. We are led from (2.24) to

$$
\left\|E-E^{h}\right\|_{r+d} \leqq \operatorname{ch}\left(\|F\|_{r}+\|\operatorname{div} F\|_{1}+\|\Delta \operatorname{div} F\|\right) .
$$

The error estimate with respect of the $L_{2}$-norm can be derived similary.

2.2. Remark. If the polygon is convex, it is possible to prove that the norms $\|\cdot\|_{r+d}$ and $\|\cdot\|_{1}$ are equivalent over $R^{0}(\Omega) \cap D(\Omega)$ (see [14] for a similar statement in $\left.\boldsymbol{R}^{3}\right)$. For such a polygon the term $\left\|\nabla \times\left(E-E^{h}\right)\right\|+\left\|\operatorname{div}\left(E-E^{h}\right)\right\|$ in $(0.2)$ can be replaced with $\left\|\nabla\left(E-E^{h}\right)\right\|$.

\section{References}

[1] BABUŠKA, I.: Finite element method for domains with corners. - Computing 6, 1970, 264-273.

[2] BABušKa, I., and M. B. RosenzWEIG: A finite element scheme for domains with corners. Numer. Math. 20, 1972, 1-21.

[3] Bers, L., and M. Schechter: Elliptic equations. - Partial differential equations edited by L. Bers, F. John, and M. Schechter, Lectures in Applied Mathematics III, Interscience Publishers, New York-London-Sydney, 1964, 131-299.

[4] Kondrat'ev, V. A.: Boundary problems for elliptic equations in domains with conical or angular points. - Trans. Moscow Math. Soc. 16, 1967, 227-313.

[5] LaAsonen, P.: On the discretization error of the Dirichlet problem in a plane region with corners. - Ann. Acad. Sci. Fenn. Ser. A I 408, 1967, 1-16.

[6] LeIs, R.: Zur Theorie elektromagnetischer Schwingungen in anisotropen inhomogenen Medien. Math. Z. 106, 1968, 213-224.

[7] LeIs, R.: Zur Theorie der zeitunabhängigen Maxwellschen Gleichungen. - Ber. Ges. Math. Datenverarb., Bonn 50, 1971,

[8] Mehra, M. L.: Zur asymptotischen Verteilung der Eigenwerte des Maxwellschen Randwertproblems. - Dissertation, Bonn, 1978.

[9] Melzer, H., and R. Rannacher: Spannungskonzentrationen in Eckpunkten der vertikal belasteten Kirchhoffschen Platte. - Sonderforschungsbereich 72, Approximation und Optimierung, Bonn, preprint no. 270, 1979. 
[10] NeitTaAnmäKI, P., and R. Picard: Error estimates for the finite element approximation to a Maxwell-type boundary value problem. - Numer. Funct. Anal. Optimization (to appear).

[11] PICARD, R.: Zur Theorie der zeitunabhängigen Maxwellschen Gleichungen mit der Randbedingung $n(\nabla \times E)=n(\nabla \times H)=0$ im homogenen, anisotropen Medium. - Bonn. Math. Schr. 65, 1973, 1-76.

[12] SaRAnen, J.: Über die Approximation der Lösungen der Maxwellschen Randwertaufgabe mit der Methode der finiten Elemente. - Appl. Analysis 10, 1980, 15-30.

[13] Saranen, J.: Über das Verhalten der Lösungen der Maxwellschen Randwertaufgabe in Gebieten mit Kegelspitzen. - Math. Methods Appl. Sci. 2, 1980, 235-250.

[14] Saranen, J.: Über das Verhalten der Lösungen der Maxwellschen Randwertaufgabe in einigen nichtglatten Gebieten. - Ann. Acad. Sci. Fenn. Ser. A.I. 6, 1981, 15-28.

[15] Stephan, E.: Conform and mixed finite element schemes for the Dirichlet problem for the bilaplacian in the plane domains with corners. - Math. Methods Appl. Sci. 1, 1979, 354-382.

[16] Strang, G., and G. J. Fix: An analysis of the finite element method. - Prentice-Hall, Inc., Englewood Cliffs, N.J., 1973.

[17] WECK, N.: Maxwell's boundary value problem on Riemannian manifolds with nonsmooth boundaries. - J. Math. Anal. Appl. 46, 1974, 410-437.

[18] WiLcox, C. H.: The steady-state diffraction of electromagnetic radiation by an obstacle in an inhomogeneous anisotropic conducting medium. - Arch. Rational Mech. Anal. 14, 1963, 326-336.

University of Jyväskylä

Department of Mathematics

Sammonkatu 6

SF-40100 Jyväskylä 10

Finland

Received 19 March 1980 